\title{
Mathematical Morphology and Binary Geodesy for Robot Navigation Planning
}

\author{
F. Ortiz, S. Puente, and F. Torres \\ Automatics, Robotics and Computer Vision Group, \\ Dept. Physics, Systems Engineering and Signal Theory. University of Alicante, \\ P.O. Box 99, 03080 Alicante, Spain \\ \{fortiz, Santiago.puente, Fernando.torres\}@ua.es
}

\begin{abstract}
A new method for obtaining the optimal path to robot navigation in 2-D environments is presented in this paper. To obtain the optimal path we use mathematical morphology in binary worlds and the geodesic distance. The navigation algorithm is based on the search for a path of minimum cost by using the wave-front of the geodesic distance of the mathematical morphology. The optimal path will be the one that minimize the direction changes of the robot. The algorithm of optimal path will be applied in several and complex 2-D environments.
\end{abstract}

\section{Introduction}

In path planning for robot navigation it is necessary to know information about the environment in order to efficiently execute the navigation tasks. The information can be represented with different abstraction levels, considering geometric and/or topological information of the environment [1]. In this paper, the approach used considers geometric information of the environment for generating a map and then, using it, the planner computes the optimal navigation path between two points of the environment.

The techniques for path generation in the environment can be divided into three categories: global, local or mixed [2]. These techniques can be used with geometric or topological information.

Global techniques need a global representation of the environment to search for a global solution to the problem, considering the full representation of the environment.

Local techniques only consider some part of the environment for planning the path, i.e., the vicinity of the search point. These techniques are less time-consuming than the global ones, but they can achieve a local minimum instead of the global one.

Mixed techniques combine the ideas of global and local techniques, obtaining good results by using the best characteristics of each technique.

Once the environment representation is made and the search technique is selected, the relevant literature offers different approaches for seeking the best navigation path for going from one point to another. Taking these techniques into consideration, we can emphasize the following ones: fuzzy logic [3], discrete artificial potential fields [4], Voronoi graphs, genetic algorithms [5], graph search optimization paths [6].

Our approach supposes a known world, represented in a 2-D geometric map. This map is transformed into a discrete world representation, using a global approach of 
the world. By using the geodesic transformations of the mathematical morphology, all the possible paths have been computed. Once these paths are obtained, we propose a method for choosing the best of these, using a branch-and-bound algorithm, taking several rules for choosing the optimal one into consideration.

In other hand, mathematical morphology is a non-linear image-processing approach, which is based on the application of the lattice theory to spatial structures. Mathematical morphology is a powerful image-analysis technique with applications in filtering, enhancement, feature extraction, etc [7,8,9].

In binary morphology, image objects are considered as sets. All the morphological operations are based on the interaction between the original image and another set of a known shape, called the structuring element. In geodesic transformations, the morphological operators applied to an original image involve a second image, known as the mask, which conditions the final results.

This paper is divided into the following sections: In Section 2 geodesic transformations of the mathematical morphology are presented. In Section 3, the rules used for the branch-and-bound algorithm are explained. In Section 4, several examples of the algorithm are shown, computing the best path in the same world and using different starting and finishing points of the trajectory. Finally, our conclusions are presented Section 5 .

\section{Geodesy and Connectivity in Mathematical Morphology}

Geodesic distance was introduced in the framework of image analysis, in 1980, by Lantuéjoul and Beucher [10] and is the base for several morphological operators [11]. The definition of the geodesic distance is as follows:

Let $A$ be a connected set. The geodesic distance $d_{A}(i, j)$ between two pixels $i$ and $j$ is defined as the length of the shortest path from $i$ to $j$. $C$ being $=\left(\mathrm{c}_{1}, \mathrm{c}_{2}, \ldots, \mathrm{c}_{\mathrm{n}}\right)$ the coordinates of the path joining $i$ and $j$, all of which are included in $A$ :

$$
d_{A}(i, j)=\min \left\{\operatorname{length}(C) \mid c_{1}=i, c_{n}=j \text { and } C \subseteq A\right\}
$$

The set $A$ is referred to as the geodesic mask. If $A$ is not connected, the two pixels $i$ and $j$ may belong to two different connected components of $A$. In this case, the geodesic distance is infinite. The geodesic distance is always greater than or equal to the euclidean distance. In Figure 1, the geodesic distance in a 2-D environment can be seen.

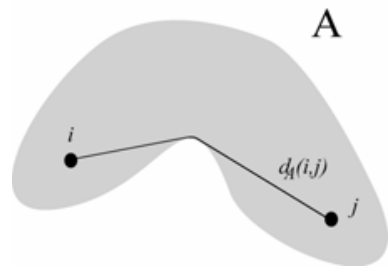

Fig. 1. Geodesic distance in a 2-D environment 
If we consider the grouping of pixels, we can calculate the geodesic distance between a point $i$ and any sub-set of pixels $X$ in $A$. In such a case, $d_{A}(i, X)$ is the shortest distance between $i$ and any point $j$ in $X$ :

$$
d_{A}(i, X)=\min _{\forall j \in X}\left\{d_{A}(i, j)\right\}
$$

Given that the geodesic distance satisfies the axioms of a metric, the distance between any sub-set $X$ in $A$ from any pixel $i$ in $A$, is $d_{A}(i, X)$.

The geodesic distance can be calculated by geodesic dilation. Indeed, the successive thresholds of the geodesic distance function of $X$ in $A$ correspond to the successive geodesic dilations of $X$ in $A$ :

$$
\delta_{A}^{(n)}(X)=\left\{i \in A \mid d_{A}(i, X) \leq n\right\}
$$

Where the geodesic dilation of size $n$ of a marker set $X$ with respect to a mask $A$ is obtained by performing $n$ successive geodesic dilations of $X$ with respect to $A$ :

$$
\delta_{A}^{(n)}(X)=\delta_{A}^{(n)}(X)\left[\delta_{A}^{(n-1)}(X)\right]
$$

The geodesic dilation of size 1 of the marker set $X$ with respect to the mask $A$ is the intersection of the dilation of the marker set $X$ with the geodesic mask:

$$
\delta_{A}^{(1)}(X)=\delta^{(1)}(X) \bigcap A
$$

\subsection{Distance and Connectivity}

Connectivity is classically studied in a topological or a graph-theoric setting $[12,13]$. With different types of connectivity between pixels, the results of the geodesic distance will be different. In flat settings, the connectivity will be either 4 or 8 . Connectivity 8 allows diagonal movements, while connectivity 4 does not. In Figure 2 we can see the different connectivity [14].

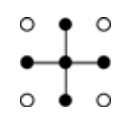

(a)

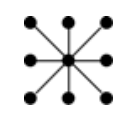

(b)

Fig. 2. Connectivity graph. 4-connectivity (a) and 8-connectivity (b)

In Figure 3, we show an example of the function of the geodesic distance in a mask from a marker set, using 4-connectivity and 8-connectivity. The grey shades of the mask represent obstacles in which the marker can not be propagated. The marker is a $3 \times 3$ set.

The main application of the geodesic distance transformation was described by Verbeek et al in [15] and Lengyel et al in [16]. By back-tracking the distance propagation, one can find the shortest path between any two points. In this article, we employ the geodesic distance for this very objective. We will move the robot from $i$ to $j$ in a 2-D environment $A$, by means the wave-front of the geodesic distance $d_{A}(i, j)$. 


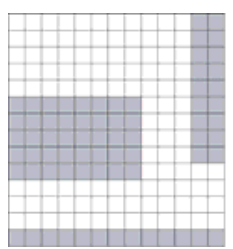

(a)

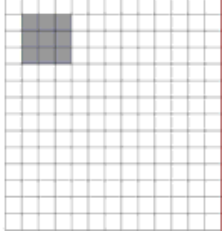

(b)

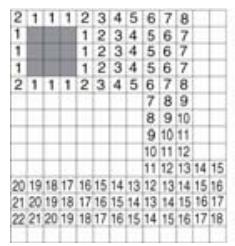

(c)

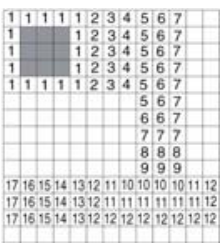

(d)

Fig. 3. Geodesic distance function in a mask (a) from a marker (b), using 4-connectivity and 8-connectivity

In this new application the marker will be a mobile robot and the mask will be the environment of movement. More than one path linking $i$ and $j$ in the environment may have the same minimal length $d_{A}(i, j)$, so that we will have to use algorithms that reduce the number of paths, based on a given movement cost in following the path.

\section{Selecting Reference Points for the Trajectory}

To accomplish a trajectory by a robot, the geodesic morphology gives us several possible solutions according to the connectivity of the discrete world used for its computation. Taking that into consideration, one of these trajectories has to be selected to be followed by the robot arm. A branch-and-bound algorithm has been used to select the best path. The characteristics used for determining the optimal path are the followings:

- Minimizing the number of changes of direction. If there are two paths with the same distance but with different amounts of changes in direction, the one with the least changes is selected as a good one (Figure 4.a).

- Selecting the shortest euclidean path, taking into account that two paths with the same number of directional changes can arrive at the same point by different ways. To resolve this problem, a linear movement, either horizontal or vertical, is associated with a weight of 5 and a diagonal movement with a weight of 7. This is because the diagonal distance between two points is $\sqrt{ } 2$ and the linear distance, either horizontal or vertical, is 1 . The computation of the square-root is more time-consuming than the use of integral numbers. The values 5 and 7 have been selected to minimize the error that arises in that approach. The best path of the two will be the one with the lower weight (Figure 4.b)

The information used for selecting the optimal path with these rules, can give us several paths with the same geodesic and euclidean costs. This happens because we are using a discrete representation of the world. The real world is not discrete, so that continuous movements can be followed, and the directions of these movements are not restricted to eight values. 
The fact of considering a continuous world is taken into consideration in the following step of the algorithm. When we have obtained a set of paths with the minimum cost, according to the previous criteria, these paths are reduced, taking into consideration that if there are several different paths to arrive at the same point, and the straight line in the continuous world between them is free of obstacles, then we can use this straight line instead of the way defined by these paths (Fig. 5).

Finally, to define the path, instead of using all the points in the discrete world, which will be impossible if we define straight lines in the continuous world, only the ones in the corners where the trajectory changes direction are stored for controlling the path. Between each pair of points the path has to be a straight line. In Figure 6 we can see a flowchart to the algorithm presented here.

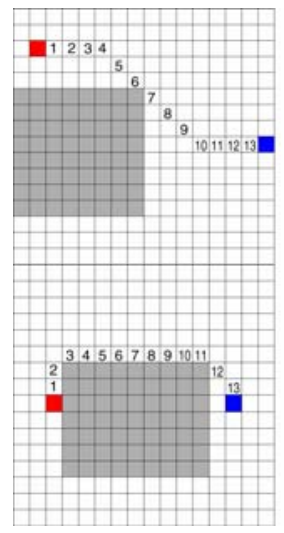

(a)

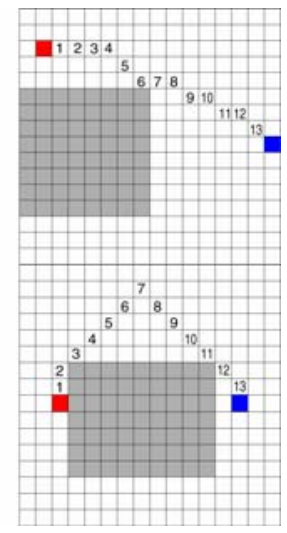

(b)

Fig. 4. (a) Several paths with the same geodesic distance (8-connectivity) and with different amounts of changes. The best one is the one in the left image. (b) Several paths with the same number of directional changes but with different euclidean costs. The best one is the one in the left image. (8-connectivity).

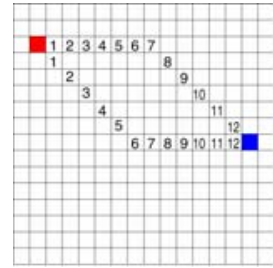

(a)

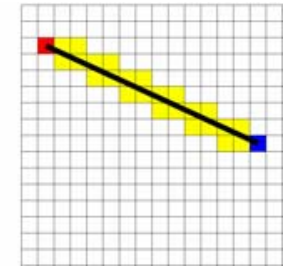

(b)

Fig. 5. (a) We can see several paths with the same cost for arriving from one point to another. (b) The best continuous path is shown, the straight line. To know whether it is possible to follow this line, the discrete positions marked in the image have to be free of obstacles. 


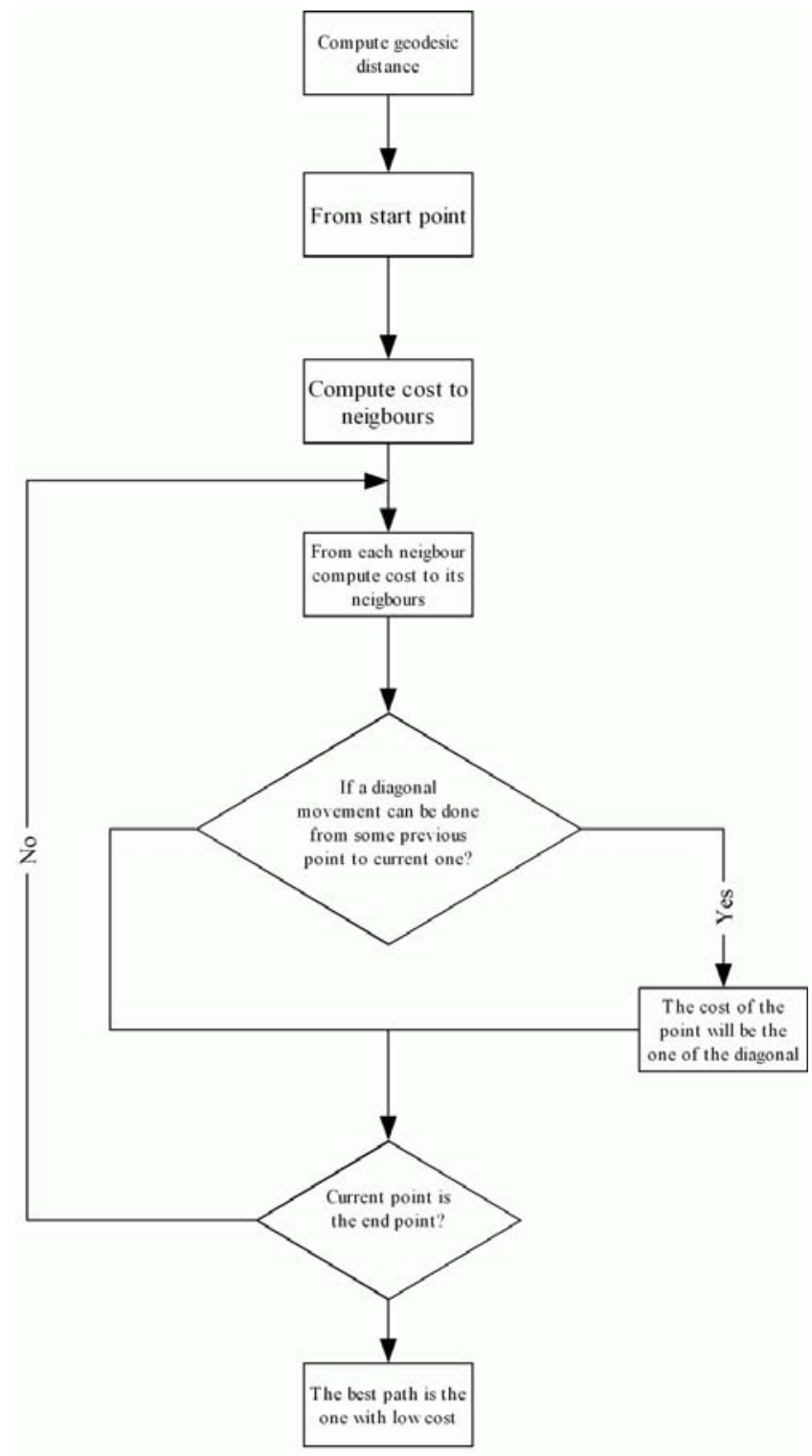

Fig. 6. Flowchart of the algorithm for robot navigation planning

\section{Geodesic Paths for Robot Navigation}

The examples for testing the algorithm use a 2-D environment, which has been converted to a discrete world. The robot (marker) is represented by a $n \times m$ set $\left(n \in \mathbb{Z}^{2}\right.$, $m \in \mathbb{Z}^{2}$ ). To reduce calculations, we dilate the obstacles and edges of the environment 
(mask) with a structuring element of size $n \times m$. This way, we only compute the geodesic distance from the centre of the marker. If we use 8-connectivity, the dilation is made with a $(n+1) \times(m+1)$ to avoid collisions.

To compute the path to be followed, the geodesic approximation and the branchand-bound algorithm are used for selecting the best path.

In Figure 7.a, we show the resulting path for going from Point $(25,25)$ to Point $(163,17)$, using an object marker (robot) of $3 \times 3$ in size. As we can see, the path followed is composed of the straight lines that join the points of reference obtained from the algorithm. In Figure 7.b, we show the resulting path for going from Point $(134,109)$ to Point $(119,109)$ using a marker of $3 \times 3$ in size. In this example, there are two possible solutions with the same cost. Both solutions are given by the algorithm.

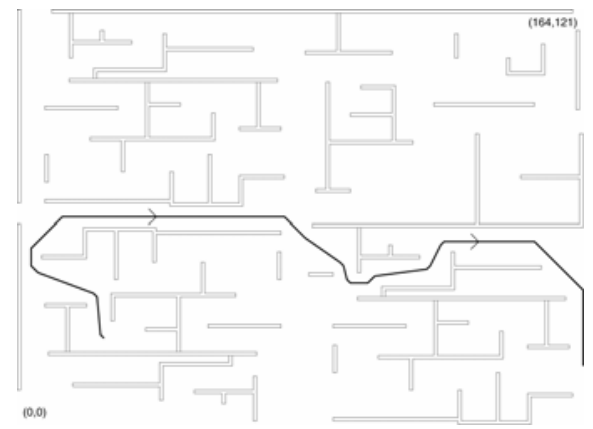

(a)

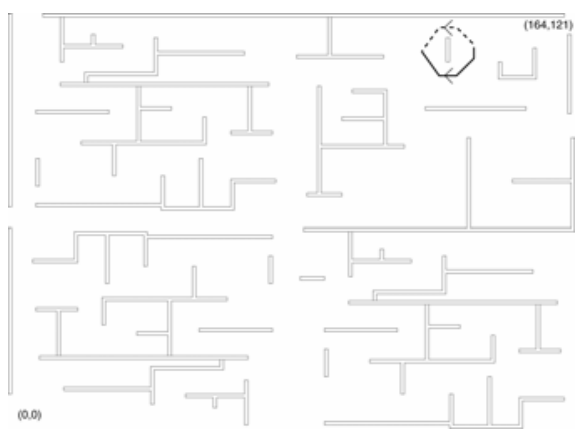

(b)

Fig. 7. (a) Path followed for going from Point $(25,25)$ to Point $(163,17)$. (b) Path followed for going from Point $(134,109)$ to Point $(119,109)$. The dotted line represents one path and the continuous line the other one.

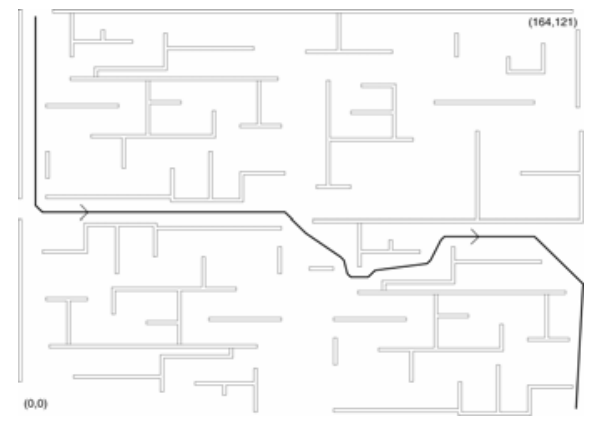

(a)

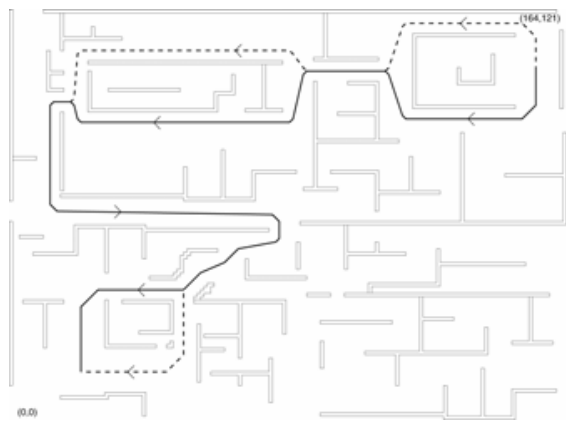

(b)

Fig. 8. (a) Path followed for going from Point $(5,118)$ to Point $(161,2)$. (b) Path followed for going from Point $(154,102)$ to Point $(21,14)$. 
In Figure 8.a, we show the resulting path for going from Point $(5,118)$ to Point $(161,2)$ using an object of $3 \times 3$ in size. In this example, there is only one possible path to be followed by the object. In Figure 8.b, we show the resulting path for going from Point $(154,102)$ to Point $(21,14)$, using an object of $3 \times 3$ in size. In this example, the environment used for computing the path is different from the one used in the previous examples, but of the same size. There are several paths for going between desired points. The first route is indicated by the continuous line, and another route is represented by the dotted line. These two routes can be combined to gives us the 8 possible routes with the minimum cost. These 8 paths have the same directional changes and the same euclidean cost.

All the examples of the new algorithm for robot navigation has been tested in a Intel Pentium 4 processor $2 \mathrm{GHz}$, and the CPU time (in seconds) has been similar to current algorithms for navigation planning: 0.5-1 seconds in above discrete worlds.

\section{Conclusions}

In this paper, we have presented a new approach for obtaining the optimal path for robot navigation in 2-D environments. The originality of this algorithm is that it is based on the geodesic distance of the mathematical morphology. We seek the paths with minimum cost. This cost is the number of directional changes in the movement. Taking that into consideration, for paths with the same cost, the one with the minimum euclidean distance in the continuous world is selected.

Several examples of the application of this algorithm have been presented. In these examples, the path obtained for going from the starting to the finishing points have been shown. It can be appreciated that the selected path uses a straight line in the continuous world, between pairs of points, and these lines do not exist in the discrete world. This is an important optimization of the algorithm proposed, which computes the path in the discrete world and gives the solution in the continuous one.

At present, we are working in the adaptation of our algorithm for a fast calculation in discrete and non discrete worlds and in a 3-D navigation. This approach in 3-D can be used for air and submarine navigation, and for computing a robot arm trajectory in a known environment.

\section{References}

1. Zavlangas, P., Tzafestas, S.: Integration of topological and metric maps for indoor mobile robot path planning and navigation. Lecture Notes in Artificial Intelligence. Vol. 2308 (2002) 121-130.

2. Reid, M.: Path Planning Using Optically Computed Potencial Fields. In: Proc. IEEE International Conference on Robotics and Automation, Atlanta (1993) 295-300.

3. Meigoli, V. Kamalodin, S., Nikravesh, Y., Talebi, H.: A new global fuzzy path planning and obstacle avoidance scheme for mobile robots. In: Proc. Advanced Robotics, Coimbra (2003) 1296-1301.

4. Zhuang, X., Meng, Q., Yin, B., Wang, H.: Robot path planning by artificial potential fields optimization based on reinforcement learning with fuzzy state. In: Proc. Intelligent Control and Automation, Shanghai, (2002) 1166-1170. 
5. Zou, X., Cai, Z., Sun, G.: Non-smooth environment modelling and global path planning for mobile robots. Journal of Central South University of Technology. Vol.10, i.3 (2003) 248-254.

6. Hwang, J., Kim, J., Lim, S., Park, K.: A fast path planning by path graph optimization. IEEE Transactions on Systems Man and Cybernetics Part A-Systems and Humans. Vol.33, i.1 (2003) 121-128.

7. Serra, J.: Image Analysis and Mathematical Morphology. Vol I, Academic Press, London (1981).

8. Serra, J.: Image Analysis and Mathematical Morphology. Vol II: Theoretical Advances, Academic Press, London (1988).

9. Heijmans, H.: Morphological Image Operators. Academic Press, New York (1994).

10. Lantuéjoul, C., Beucher, S.: Geodesic distance and image analysis. Mikroskopie.Vol.37 (1980) 138-142.

11. Soille, P.: Morphological Image Analysis. Principles and Applications, Springer-Verlag, Berlin (1999).

12. Dugundji, J.: Topology. Allyn \& Bacon, Boston, MA (1966).

13. Diestel, R.: Graph Theory. Springer-Verlag, New York (1997).

14. Ortiz, F.: Procesamiento Morfológico de Imágenes en Color. Aplicación a la Reconstrucción Geodésica. In: Virtual Library Miguel de Cervantes www.cervantesvirtual.com, Alicante (2002) 121-136.

15. Verbeek, P., Dorst, L., Verwer, B., Groen, F.: Collision avoidance and path finding through constrained distance transformation in robot state space. In: Proc. Intelligent Autonomous Systems, Amsterdam (1986) 634-641.

16. Lengyel, J., Reichert, J., Donald, B., Greenberg, D.: Real-time robot motion planning using rasterizing. Computer Graphics. Vol.24, i.4 (1990) 327-335. 